\title{
Role of Fungal Biomass in N-Hexane Biofiltration
}

\author{
Mena M. Botros', Ashraf Aly Hassan², George A. Sorial ${ }^{3}$ \\ ${ }^{1}$ The Ohio State Wexner Medical Center, Columbus, OH, USA \\ ${ }^{2}$ Civil Engineering Department, University of Nebraska Lincoln, Lincoln, NE, USA \\ ${ }^{3}$ Department of Biomedical Chemical, and Environmental Engineering, University of \\ Cincinnati, Cincinnati, OH, USA \\ Email:ahassan@unl.edu
}

How to cite this paper: Botros, M.M., Hassan, A.A. and Sorial, G.A. (2017) Role of Fungal Biomass in N-Hexane Biofiltration. Advances in Microbiology, 7, 673-688. https://doi.org/10.4236/aim.2017.710053

Received: September 3, 2017

Accepted: October 13, 2017

Published: October 16, 2017

Copyright $\odot 2017$ by authors and Scientific Research Publishing Inc. This work is licensed under the Creative Commons Attribution International License (CC BY 4.0).

http://creativecommons.org/licenses/by/4.0/

\begin{abstract}
The biofiltration of $n$-hexane is studied to optimize determinants factors of hydrophobic VOC filtration efficiency. Four trickle-bed air biofilters (TBABs) were employed; two of which were supplied with nutrients buffered at a neutral $\mathrm{pH}$, while another two at an acidic $\mathrm{pH}$ of 4 to induce and enhance fungal growth. The loading rate of $\mathrm{n}$-hexane was kept constant in all TBABs at 13 $\mathrm{g} / \mathrm{m}^{3} / \mathrm{h}$. At each $\mathrm{pH}$ levels studied, the biomass of the TBABs was pre-acclimated using different ratios of $n$-hexane and methanol. The fungal biomass responsible for the degradation of $n$-hexane was then examined and quantified. Dichloran Rose Bengal Chloramphenicol agar was used for fungi quantification, and optical microscopy for classification. Effluent biomass was validated by measuring volatile suspended solids. Fungal counts resulting from n-hexane biodegradation were related to nitrate and carbon consumption. It was found that $\mathrm{n}$-hexane elimination capacity closely followed biomass growth, and reached a steady-state at an optimum biomass density of roughly $3000 \mathrm{cfu} / \mathrm{ml}$. Major shifts in fungal species were observed in all TBABs. Dominant fungal species grew slowly to become the most numerous, and were found to provide maximum elimination capacity, although TBABs pre-acclimated to higher methanol concentrations took less time to reach this steady-state. It was concluded, therefore, that steady and monitored growth of TBAB biomass is an essential factor in maximizing fungi's ability to metabolize VOCs and that a new ecological biofiltration model may be the most effective at VOC purification.
\end{abstract}

\section{Keywords}

Biofiltration, N-Hexane, Trickle Bed Air Biofilter (TBAB), VOCs, Fungi 


\section{Introduction}

Utilization of volatile organic compounds (VOCs) has been, and continues to be, prevalent in industry. Uses of VOCs range from the manufacturing of plastics and rubber, to the use of these compounds as solvents for industrially manufactured adhesives, and a variety of different products [1]. One such VOC, $\mathrm{n}$-hexane, is a common raw material for the formulation of shoe adhesives, leather products, and roofing. Hexane is an essential element in cooking oils extraction from seeds, for cleansing and degreasing, and in textile manufacturing [1]. Although the utilization of n-hexane is common, it has been found to be hazardous to human health. Studies have shown that through inhalation, repeated percutaneous absorption, and ingestion of $n$-hexane contaminated water or food, both humans and animals may develop cancer and neuropathy [1] [2] [3] [4]. To address this issue, the Clean Air Act was passed in order to curtail the production and release of hexane and several hundred other VOCs; however, pollution and contamination remain an issue as industries struggle to effectively and efficiently purify VOC byproduct waste.

There are several traditional methods of VOC reduction, which include incineration, adsorption, and catalytic oxidation [3] [5]. Recently, biofiltration has become a more viable option for VOC treatment, as it provides an environmentally friendly and low-cost alternative to current industrial methods. Biological systems work best for the treatment of large volumes of off-gases that contain low concentrations of biodegradable contaminants [6] [7]. Several studies highlighted the dependency of successful biofiltration on Henry's law constant, meaning that the ability of microorganisms to metabolize VOCs is significantly dependent upon VOC solubility [8] [9]. For this reason, hydrophobic VOCs such as $\mathrm{n}$-hexane present a unique challenge as they are not easily captured for metabolism by microorganisms growing on biofilter mediums.

Recent research has been committed to increasing the removal efficiency of biofiltration of hydrophobic compounds [5] [10]-[15]. Van-Groenestijn [16] was the first to show the role of fungi as a micro-organism consortium that has the ability to improve the performance of biofiltration. The study referred to several reasons for supremacy of fungi over bacteria, as they are more resistant to acidification and drying out; this, however, does not necessarily count as an advantage in biofiltration systems such as Trickle Air Bed Biofilters (TBABs), as these variables are often controlled in such systems. In addition, aerial mycelia of fungi form a larger surface area in the gas phase than bacterial biofilms, which may facilitate the uptake of hydrophobic volatile compounds, thereby overtaking the rate limiting step. Since then several studies have come to conclude that fungi are correlated with increased VOC metabolization, both hydrophobic and otherwise [14]. As such, many have attempted to increase the efficiency of these fungal biofilters [17] [18] [19], as well as characterize the traits that make these fungi more efficient at metabolizing VOCs [11] [12] [20] [21].

Knowledge concerning biofilter's microbial ecology continues to be limited 
[6] [7]. Although many studies have sought to analyze microbial functionality as due to structure, limited work has been done with the interaction of these microbes within a biofilter. Most of the studies have largely used single or double inoculums of specific isolated fungi [22] [23] [24] [25]. A few, however, utilize a microbial consortium [11] [12] [21]. For the most part, these studies have concluded varied effects on VOC elimination capacity due to the utilization of a microbial consortium. However, all indicate changes in biomass diversity with respect to time and changes to biofilter conditions. It is these same changes and the possible correlations between microorganisms and biofiltration efficiency that this study also sought to elucidate.

Previous investigations at our research group successfully increased the removal of $n$-hexane by addition of surfactants, or other hydrophobic compounds like benzene that may be mixed in with target VOCs [10]. TBAB efficiency was increased by operating the biofilters at an acidic $\mathrm{pH}$ [26]. All the previous studies were performed with a mixed micro-organism culture obtained by seeding the $\mathrm{TBAB}$ with wastewater to ensure the reproducibility of the experiments. In this study, the microbial fungi culture of four TBABs operating under different conditions to remove $\mathrm{n}$-hexane was characterized and quantified. The experimental plan was designed to operate four TBABs to remove a mixture of methanol and n-hexane with ratios of $70 \%$ to $30 \%$ and $80 \%$ to $20 \%$ by VOC volume, respectively, at neutral (7) and acidic (4) pHs [27]. Later, methanol was removed from the influent feed and fungi cultures responsible of removing $n$-hexane as a single feed was studied. This study sought to understand the relative ecosystem established by a complex community of fungi in TBABs. In addition to attempting to characterize the overall fungal community and factors that may induce additional VOC removal efficiency, general fungal morphology was also characterized at different stages of the filter ecosystem's development.

\section{Materials and Methods}

\subsection{Trickling Bed Air Biofilter}

Experiments were conducted in a TBAB, a cylindrical encasing of growth media which was supplied with nutrients to support fungal biomass. The four TBABs utilized consisted of seven cylindrical glass sections connected and sealed in series with an internal diameter of $7.6 \mathrm{~cm}$ and a total length of $130 \mathrm{~cm}$. All seven sections were filled with pellets of diatomaceous earth biological support media to a depth of about $60 \mathrm{~cm}$ (Celite ${ }^{ø} 6 \mathrm{~mm}$ R-635 Bio-Catalyst Carrier; Celite Corp., Lompoc, CA, USA) to support growth of biomass. Conditions were as follows: temperature was held constant at $20^{\circ} \mathrm{C}$, liquid was always passed downward, direction of gas flow alternated between weeks, the air flow was set up at the rate of $1.36 \mathrm{~L} \cdot \mathrm{min}^{-1}$ with a corresponding empty bed retention time (EBRT) of $120 \mathrm{~s}$, and nutrient solution was supplied at a rate of $2.0 \mathrm{~L} \cdot \mathrm{day}^{-1}$; the composition of the nutrient solution is similar to that reported in an earlier study [10]. Two TBABs "A" and "B" were operated at $\mathrm{pH} 7$ buffered by calcium carbonate, 
while both TBABs "C" and "D" were buffered at $\mathrm{pH} 4$ using sodium formate. Figure 1 illustrates the aforementioned structure and utilization of these TBABs.

Two VOCs were utilized in this study; n-hexane was obtained from Fisher Scientific (Fair Lawn, N.J) with 95\% purity, while methanol was obtained from Tedia (Fairfield, $\mathrm{OH}$ ) with $99.9 \%$ purity. Henry's law constant was reported for n-hexane at $20^{\circ} \mathrm{C}$ by different authors as $0.883 \mathrm{~atm} \cdot \mathrm{m}^{3} / \mathrm{mol}$ [28] and 0.014 atm. $\mathrm{m}^{3} / \mathrm{mol}$ [29], while methanol's was reported at the same temperature as 4.55 $\times 10^{-6} \mathrm{~atm} \cdot \mathrm{m}^{3} / \mathrm{mol}$ [30]. In the first phase the influent $\mathrm{n}$-hexane concentration was kept constant at $125 \mathrm{ppmv}$ while the methanol concentration was varied at both $\mathrm{pH}$ levels studied. On the second phase methanol was removed from the feed and n-hexane as a single VOC was introduced in the influent feed at the same influent concentration of $125 \mathrm{ppmv}$ and the same $\mathrm{pH}$. Removal efficiency of TBAB was calculated according to Equation (1).

$$
\text { Removal Efficiency }(\%)=\left(C_{\text {in }}-C_{\text {out }}\right) / C_{\text {out }} \times 100
$$

Biomass control techniques were employed in TBABs on a weekly basis to control biomass density, and thereby maintain VOC elimination capacity. In the first stage, where methanol concentration was relatively high as compared to n-hexane, backwashing technique was utilized. Afterward, stagnation technique in addition to weekly flow-switching was utilized. The details of the biomass control techniques are explained below.

Flow switching. This experimental strategy involves switching the direction of the gas flow once weekly, i.e., for a 3-week period, the direction of the gas flow for the first week was co-current with the nutrient liquid flow downwards then countercurrent on the second week upwards and finally co-current on the third week downwards.

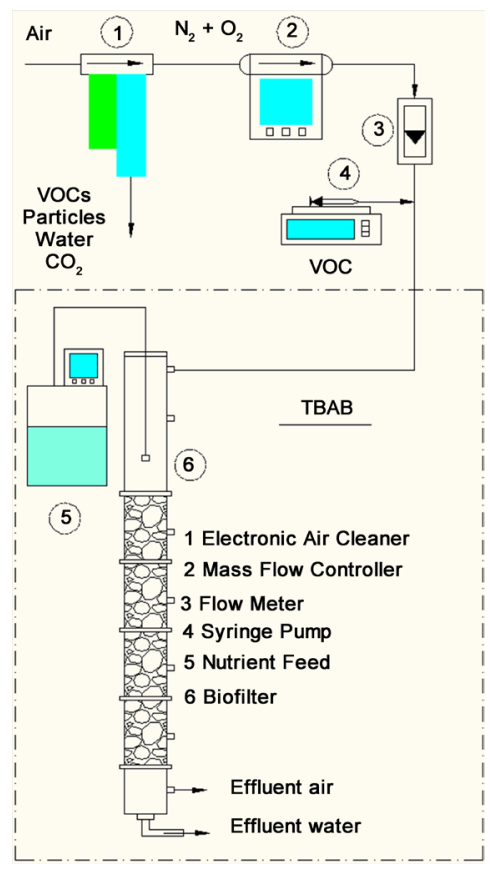

Figure 1. Schematic of the TBAB. 
Backwashing. Backwashing is only conducted if the TBAB if offline by recycling $18 \mathrm{~L}$ of buffered nutrient solution through the column for 1 hour per week. Compressed air was used when necessary to shear off the excess biomass.

Stagnant period. This experimental strategy reflects no flow (VOC, nutrient, and air) passing through the TBAB. The duration and frequency for this strategy were 2 days per week.

\subsection{Analytical Techniques}

The concentrations of VOCs in the gas phase were measured by using a gas chromatograph (GC) (HP 5890, Series II: Hewlett- Packard, Palo Alto, CA, USA) equipped with a flame ionization detector (FID) and 30-m length, 0.25-mm I.D., $0.25 \mu \mathrm{m}$ film thickness narrow bore column (DB 624, J and WK Scientific, Folsom, CA). The GC oven was programmed isothermal at $120^{\circ} \mathrm{C}$. The carrier gas $\left(\mathrm{N}_{2}\right)$ flow rate was set at $8 \mathrm{~mL} / \mathrm{min}$. The flame ionization detector was used with $\mathrm{N}_{2}$ make-up gas at a flow rate of $20 \mathrm{~mL} / \mathrm{min}$, a fuel gas flow $\left(\mathrm{H}_{2}\right)$ of $30 \mathrm{~mL} / \mathrm{min}$, and an oxidizing gas flow (air) of $300 \mathrm{~mL} / \mathrm{min}$. The detector temperature was $250^{\circ} \mathrm{C}$. Effluent gas phase sample for $\mathrm{CO}_{2}$ analysis were also taken by using gas-tight syringes through sampling ports in the TBAB. A GC (HP 5890, Series II, Hewlett-Packard) equipped with a thermal conductivity detector (TCD) was used for determining the $\mathrm{CO}_{2}$ concentrations in the effluent gas phase. The detection limit was 0.001 volume $\% \mathrm{CO}_{2}$. Detailed description of the analytical method is provided elsewhere [10]. Liquid phase samples were analyzed for $\mathrm{NO}_{3}-\mathrm{N}$, total carbon (TC), inorganic carbon (IC), and volatile suspended solid (VSS) concentration, which are the remaining concentrations of biomass found in the effluent, or ejected waste, samples. $\mathrm{NO}_{3}-\mathrm{N}$ was determined according to Standard Methods [31] 4500- $\mathrm{NO}_{3}$ - by using a Shimadzu UVmini 1240 UV_visible spectrophotometer (Shimadzu Corp., Tokyo, Japan). TC and IC were determined by using a Shimadzu TOC 5050 analyzer according to Standard Methods 5310 [31]. The VSS concentrations in the effluent and backwashing water were determined according to the same standards.

Active biomass concentrations of fungi and yeasts were also selectively estimated using plate counts on Dichloran Rose Bengal Chloramphenicol (DRBC) agar, enumerated five days after incubation at $23^{\circ} \mathrm{C}$. Effluent samples containing washed out active biomass were used as the inoculums, and were plated in multiple concentrations to attain viable plate counts. Within the first stage of biofiltration with n-hexane and methanol, effluent samples were diluted using $1.25 \mathrm{ml}$ phosphate buffer solution, $5 \mathrm{ml} \mathrm{MgCl}$ solution and $1 \mathrm{~L} \mathrm{DI}$ water. Using this diluting solution, the effluent from each filter was prepared in 1:10, 1:20, and 1:50 dilutions. Three samples of each dilution was prepared from each TBAB and plated twice a week, thereby creating 72 samples a week. Subsequently, in the second stage of biofiltration with $\mathrm{n}$-hexane, dilutions became unnecessary due to reduced biomass concentrations. In second stage biofiltration, each effluent sample from each TBAB was plated in the following volumes: $0.1 \mathrm{ml}, 0.2 \mathrm{ml}$, and $0.5 \mathrm{ml}$. Each volume, as with the dilutions, was replicated thrice for each effluent 
sample from each TBAB. Enumeration and classification according to colony morphology took place after the five day incubation period at $23^{\circ} \mathrm{C}$. After enumeration had taken place, isolation of each species was done using DRBC agar. The colonies were then analyzed using a simplified slide culture technique. Light microscopy (Nikon Eclipse E800 with Hamamatsu 3CCD Cooled Digital Camera using C7780 Image Pro Plus V6.2 Software) was utilized in examining and photographing isolated species for determination of colony morphology.

\section{Results and Discussions}

\subsection{TBABs Performance}

All four TBABs were operating at a constant $\mathrm{n}$-hexane influent concentration of $125 \mathrm{ppmv}$ corresponding to $13.2 \mathrm{~g} / \mathrm{m}^{3} / \mathrm{hr}$, but had different acclimation earlier with different $\mathrm{n}$-hexane-to-methanol influent concentrations (see Table 1). Two TBABs " $\mathrm{A}$ " and " $\mathrm{B}$ " were fed by a nutrient solution buffered at $\mathrm{pH} 4$, while "C" and " $\mathrm{D}$ " had a nutrient feed buffered at neutral $\mathrm{pH}$. Generally, acidic conditions had a positive impact on the TBAB performance. The removal efficiencies measured were: $92 \%, 93 \%, 35 \%$ and $79 \%$ for TBABs "A", "B", "C" and "D", respectively. At acidic environment, the pre-acclimation did not have an impact on the TBAB removal efficiency. The removal efficiency reported for both TBABs is higher than removal efficiency reported earlier by the authors of $82 \%$ [32]. Both TBABs took an acclimation period of 14 days to reach steady state. TBAB "B", which was acclimated with higher influent concentration of methanol, was very stable and steady while TBAB " $A$ " was fluctuating throughout the study period.

At neutral $\mathrm{pH}$ without methanol acclimation the authors reported an average removal efficiency of $43 \%$ [32]. This shows that methanol acclimation could have positive effect as in TBAB " $D$ " or negative effect as in TBAB " $\mathrm{C}$ " according to the mixing ratio. $\mathrm{TBAB}$ " $\mathrm{D}$ " had similar behavior to both " $\mathrm{A}$ " and " $\mathrm{B}$ " regarding the 14 days acclimation period, while $\mathrm{TBAB}$ " $\mathrm{C}$ ” performance was fluctuating very rapidly reaching $80 \%$ just after stagnation period and dropping down to almost $0 \%$ by the end of the week. TBAB "C" was over saturated with live microorganisms. This had a very negative impact on the performance.

Table 1. Operating conditions for all TBABs at the first stage.

\begin{tabular}{cccccccc}
\hline TBAB & $\begin{array}{c}\text { Influent Concentration } \\
\text { (ppmv) }\end{array}$ & $\begin{array}{c}\text { VOCs Ratio } \\
(\% \text { by } \\
\text { volume })\end{array}$ & $\mathrm{pH}$ & $\begin{array}{c}\text { Loading } \\
\text { Rate } \\
\left(\mathrm{g} / \mathrm{m}^{3} / \mathrm{h}\right)\end{array}$ & $\begin{array}{c}\text { Maximum } \\
\text { Elimination } \\
\text { Capacity }\end{array}$ & $\begin{array}{c}\text { Maximum } \\
\text { n-hexane } \\
\text { Removal } \\
\text { Efficiency } \%\end{array}$ \\
\cline { 2 - 5 } A & 125 & 975 & $80: 20$ & 4 & 13.2 & 12.9 & 92 \\
B & 125 & 1668 & $70: 30$ & 4 & 13.2 & 12.9 & 93 \\
C & 125 & 1668 & $70: 30$ & 7 & 13.2 & 12.9 & 35 \\
D & 125 & 975 & $80: 20$ & 7 & 13.2 & 12.8 & 79 \\
\hline
\end{tabular}




\subsection{Patterns in Biomass}

As aforementioned, VSS was utilized as a means of measuring both viable and dead biomass in the effluent, which again is representative of the biomass within the filter itself. Although this presented interesting patterns, which will be discussed later, we sought to concentrate solely on the viable biomass within the filter using plate counts to determine what trends, if any, were perceptible. Therefore, after identification of main cultured strains, each strain was quantified over the operation time for each filter and analyzed.

There were several trends shared by each of the filters. Firstly, overall biomass in the filters generally increased over time. Secondly, the same six species were cultured from each of the filters and were found to be the main species, or the ones with the highest viable counts. Therefore, we elected to follow these species as they were likely to have the most impact in the filter. The fungi were identified using microscopy, and were classified according to genus. The six identifications made included: Actinomadura, Aureobasidium, Cladosporium, Rhodotorula, Geotrichum, and Lecythophora. Population densities of each species changed over time, such that each species exerted dominance in the filter at a point in time prior to reaching steady-state where the maximum and constant removal efficiency of the filter was attained.

TBAB A: Beginning at an average of $413 \mathrm{cfu} / \mathrm{ml}$ and reaching a measured maximum of $3016 \mathrm{cfu} / \mathrm{ml}$, Filter A, as with all other filters, had a general increase in biomass over the period of time allotted for filtration. The species most visible at the beginning of filtration, from highest population density to lowest, were Cladosporium, Geotrichum, followed by equal amounts of Aureobasidium and Lecythophora. With time, these species declined in number, and the Actinomadura was to come into dominance, followed lastly by the Rhodotorula. These results are represented in Figure 2, which shows the decline of the initial four species, and Figure 3, which illustrates the intermittent species dominance and eventual dominance by Rhodotorula.

TBAB B: Similar patterns in individual species counts were observed in TBAB $\mathrm{B}$ with the following exceptions. In TBAB B the Rhodotorula species reached its maximum concentration much sooner than in TBAB A. In week four, this species was hardly detectable in TBAB A, while in TBAB B, the species had reached a concentration of roughly $3000 \mathrm{cfu} / \mathrm{ml}$; this, however, would also be the highest concentration it would achieve, and the population density soon after fell dramatically, as compared to TBAB A, which saw an increasing trend in this species population density. Secondly, the species of Actinomadura was much more prevalent in this TBAB, than in TBAB A. A maximum concentration of $60 \mathrm{cfu} / \mathrm{ml}$ was attained for this species in TBAB $A$ in week four of operation, whereas in TBAB B a concentration of $225 \mathrm{cfu} / \mathrm{ml}$ was attained in the same week. In addition, there was an increasing trend in its population density, reaching a maximum in the final week of operation of roughly $2205 \mathrm{cfu} / \mathrm{ml}$. These differences in species density may also account for differences in TBAB performance, as will be noted later. 


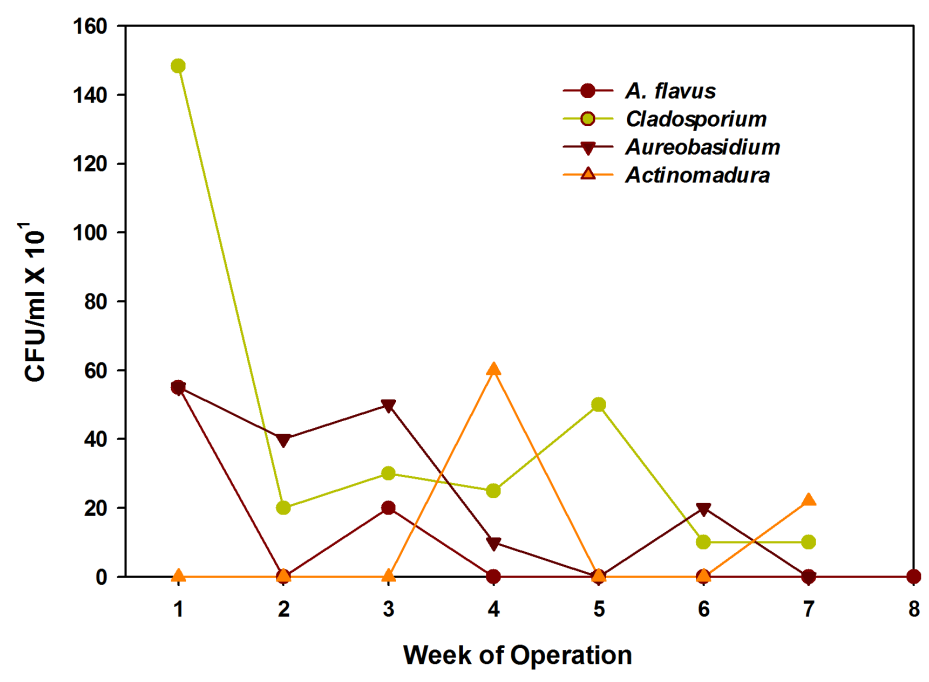

Figure 2. Colony counts, in $\mathrm{cfu} \cdot \mathrm{ml}^{-1}$, per introductory species in TBAB A during 8 weeks of operation following acclimation period.

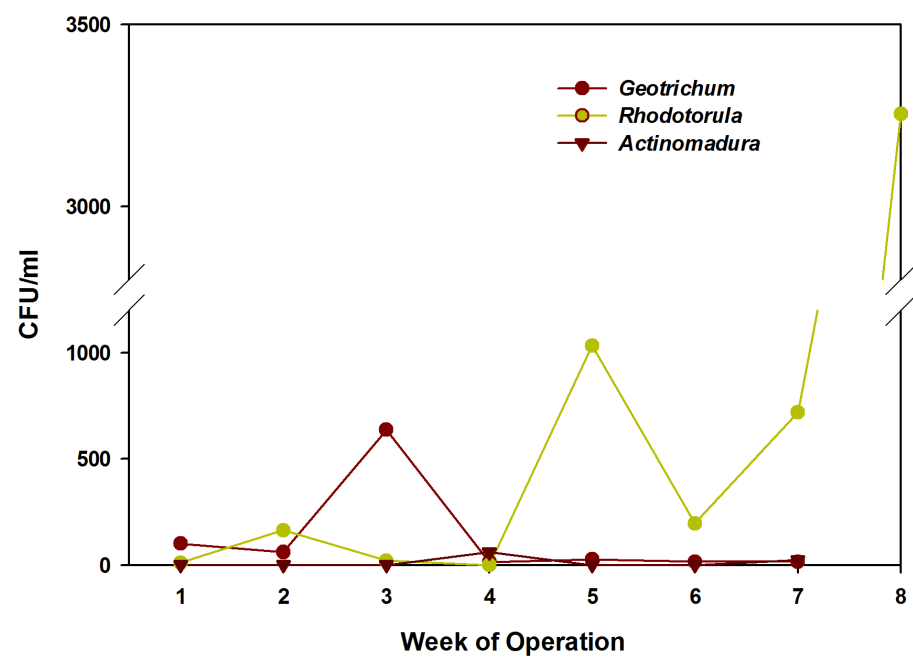

Figure 3. Colony counts, in $\mathrm{cfu} \cdot \mathrm{ml}^{-1}$, per final dominant species in Filter A during 8 weeks of operation following acclimation period.

TBABs C \& D: Similar patterns in individual species counts were observed in these TBABs as with TBAB A.

Overall Comparison: Other than a general increase in overall biomass, no correlations could be derived between filters and overall viable counts. Patterns were easily seen, however, between filters when utilizing VSS and individual species counts.

When comparing individual species counts, changes in dominance were observed in all filters, with largely decreasing viable biomass within the first two weeks of operation, followed by intermittent dominance by a variety of species. Across all filters, Cladosporium had the largest initial colony count and decreased in concentration with time. Also, Rhodotorula was observed to be the last species to take dominance within the TBABs, but by a much larger majority, or biomass concentration, than any other species previously observed. Lastly, all 
other species previously listed had intermittent dominance.

The overall longitudinal relationship between VSS and colony counts were weak with an $\mathrm{R}^{2}$ minimum of 0.4 . While CFUs are a quantitative measure of viable cells, VSS combine both viable and dead cells. The variability in dead cells amount seemed to cause the weak relationship. However, when comparing specific time periods within the operation of the filters, both strong positive and negative correlations could be detected, as best depicted by Figure 4(a) and Figure 4(b) illustrating the relationship between viable counts and VSS in Filter B. When negative correlations were observed, viable biomass, as determined by colony counts, were always decreasing. Although viable biomass increased generally over time, different species took dominance at different times; in doing so, one or more species would decline in population density, leading to a more immediate decreased reading in viable biomass. VSS in all filters was generally increasing over time, as viable biomass was generally increasing as well as the accumulation of dying biomass, hence the inverse relationship. In contrast, when viable biomass was increasing, or a new species was coming to take dominance, a positive correlation would exist. These changes in periods of positive and negative correlation between viable biomass (colony counts) and VSS were found to coincide with changes in dominance in each filter. Thus, it was found that this came to be another indicator of a unique ecological environment within each of the TBABs.

\subsection{Identification and Morphology}

As previously mentioned, changes in species dominance was observed via plate counts. In order to better understand why these changes were taking place within the TBABs, microscopy was utilized to identify and characterize the fungi cultured. Identification was completed using several published texts [33] [34], which provided means of identification via morphological features. As to avoid error, species were identified according to genus, rather than species level. Morphology was also noted and compared to species counts.

Records of colony types according to morphology indicated an obvious change in primary or dominant species present in the filter over time. Using microscopy, each of these seven colony types, which could be cultivated on DRBC agar, were examined. It was found that species present initially in the filter at highest concentrations, relative to other species, did not maintain that dominance over time. Although these and later dominant species could be found at any point in time within the filter, those present in initial stages had a largely different morphology than those in later stages of the experiment.

Many previous studies have indicated that it is most likely fungi that aid in the capture of VOCs is due to their chitin based hyphae and conidiophores. However, in a filter selected for fungal development, we did not see this morphology gain constant dominance. Rather, in later stages of biofiltration, yeast-like colonies were the most prevalent within the TBAB as seen in the images provided in Figure 5. 


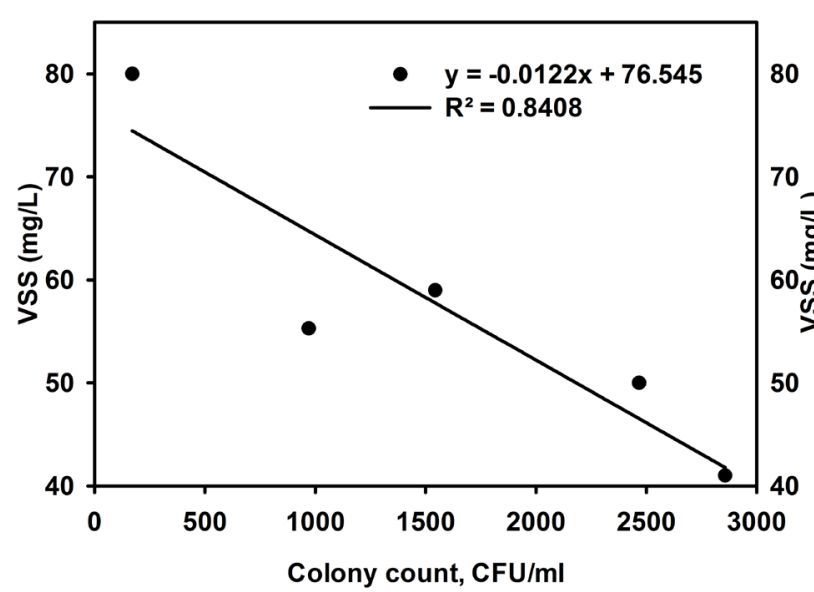

(a)

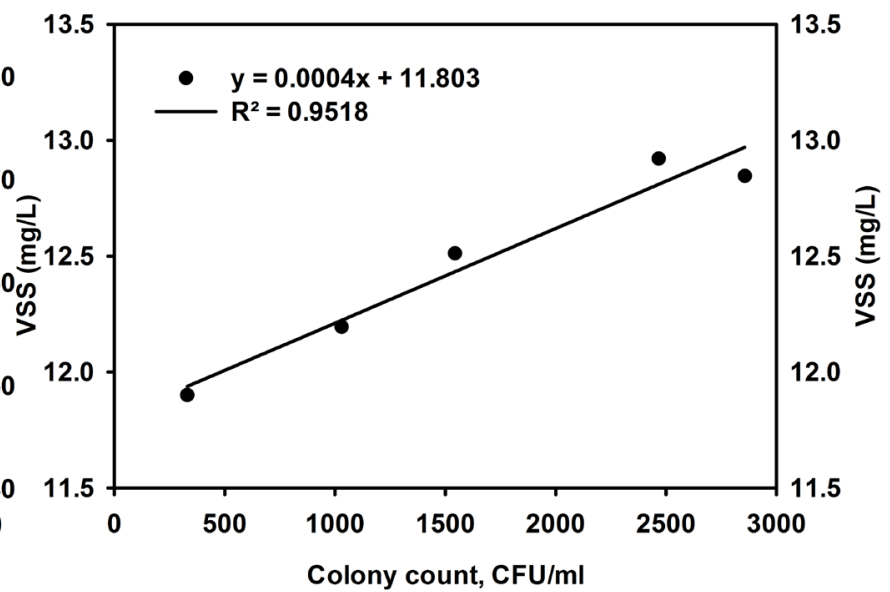

(b)

Figure 4. Correlation between colony counts, in $\mathrm{cfu} \cdot \mathrm{ml}^{-1}$, in comparison to VSS in Filter B (a) during first two weeks of operation following acclimation period (negative correlation) (b) during first two weeks of operation following acclimation period (positive correlation).
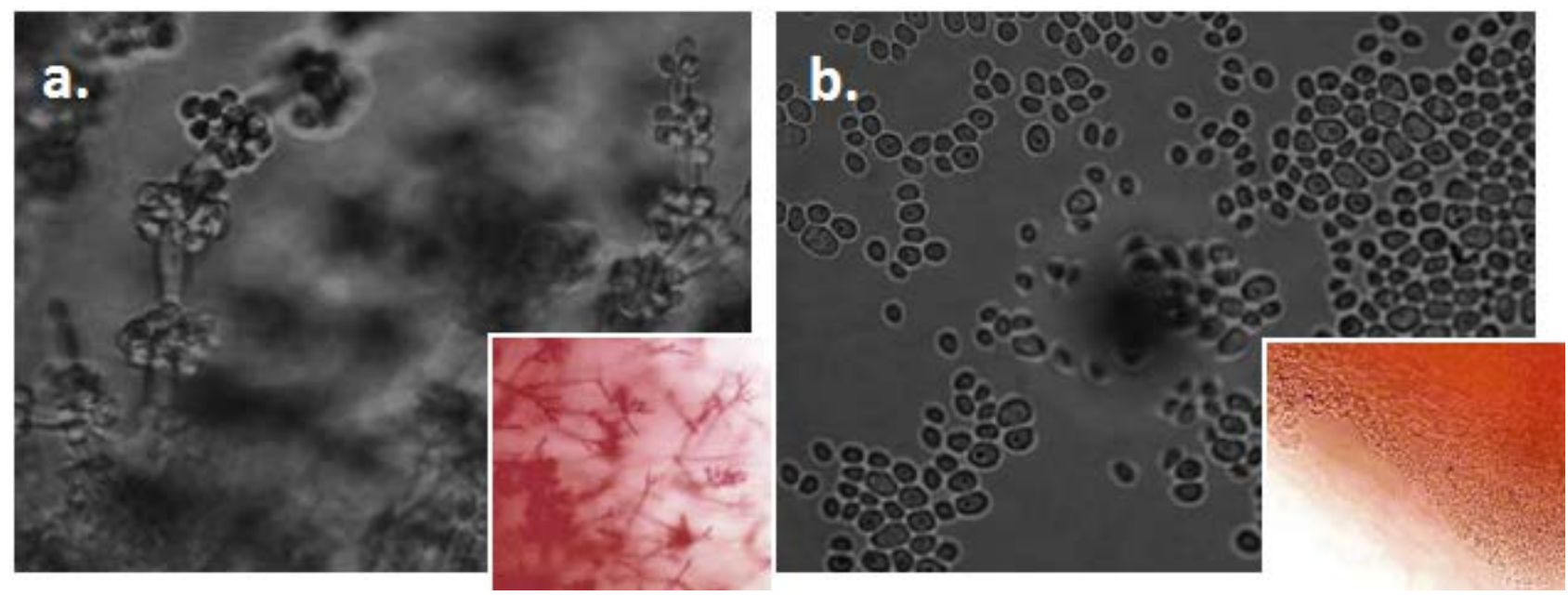

Figure 5. Cladosporium, an early successional species, and Rhodotorula, a later successional species, isolated from Filter (a) Larger images taken at $100 \times$ and smaller images to the forefront taken at $10 \times$ magnification, respectively.

According to these observations in colony morphology, the following was concluded; in initially, the biomass requires higher surface area in order to capture VOCs, which it uses as a carbon and energy source. Hyphae, long filamentous structures of fungi, help to establish this higher surface area. This hyphae acts like a sort of net, allowing the capture of the VOCs for metabolization, or in terms of the industry, VOC purification. The first successional species that grew in this TBAB seeded with a variety of organisms, were ones that are the most efficient at capturing and metabolizing the VOCs.

Later successional species, as with those in facilitative ecological models, come to succeed due to the environment established by the preliminary species. As indicated by the lack of correlation of VSS and colony enumeration, most likely developed from the left over or shared carbon material and consumption of dead biomass. Increases in VSS and decreases in viable colony count at certain stages 
of biofiltration suggested that the next dominant species outgrew the previous species, later utilizing its organic matter, as again indicated by increased viable counts and decreased VSS shortly thereafter. These species do not seem to require such an extensive hyphal structure. Rather, it seems that once established in higher numbers, these species are approximately as effective as the earlier successional species were in smaller numbers, or a lower population density. Thus, the overall shift in species dominance changed over time, but was eventually able to maintain maximum n-hexane elimination capacity.

\subsection{Relationship between CFU and Different Operating Parameters}

Colony counts were investigated against several operating parameters. The increase in CFUs means that the amount of viable cells in the effluent liquid is increasing. It is worthwhile to mention that the effluent liquid volume is relatively small, ca. 2 L/day. Comparing the elimination capacity with the corresponding CFU sheds light on the acclimation procedure of TBABs. During operation, all TBABs showed the same relationship; there is a sharp increase in the elimination capacity while the colony count is almost stable. After reaching the maximum elimination capacity the CFUs start to increase until the optimum CFUs is reached. Figure 6 shows the change of elimination capacity vs. CFU/ml.

As seen in Figure 6, all filters came to stabilize around an elimination capacity of $13 \mathrm{~g} / \mathrm{m}^{3} \mathrm{~h}$ after steady state (optimum biomass concentration with the natural progression of species dominance over time) was achieved. This is a significant achievement as each TBAB had a loading rate of $13.23 \mathrm{~g} / \mathrm{m}^{3} \mathrm{~h}$, meaning each filter achieved a very high VOC removal efficiency.

CFUs have a proportional relationship with some variables measured in the liquid phase like volatile suspended solids (VSS), nitrate utilization and inorganic carbon. The relationship between viable biomass ( $\mathrm{cfu} / \mathrm{ml}$ ) counts and VSS was previously mentioned. Similar proportional relationships were found between CFUs and daily nitrate utilization, as well as inorganic carbon in the effluent samples. As was expected, an increase in nitrate utilization was observed with an increase in viable biomass, thereby creating a positive correlation between the two factors across all TBABs. The increase in nitrate utilization is therefore a direct indication of enhanced biodegradation and accordingly the elimination capacity.

On the other hand, strong relationship with a minimum $\mathrm{R}^{2}$ of 0.9 was found between CFUs and inorganic carbon as is seen in Figure 7. Inorganic carbon, like nitrate utilization, indicates the biodegradation activity. This is because inorganic carbon detected in the effluent most often takes the form of dissolved carbon dioxide, a byproduct of any hydrocarbon metabolization. Such a relationship suggests that inorganic carbon and nitrate utilization measurements are interchangeable. Additionally, this provides evidence that increased removal efficiency and elimination capacity across all TBABs is due solely to the increased 


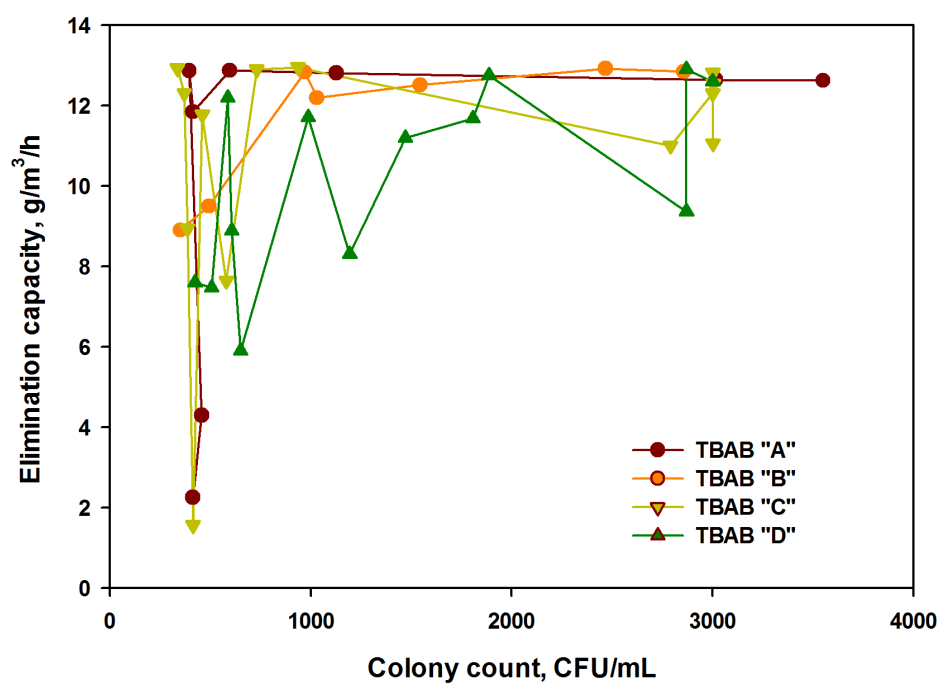

Figure 6. n-Hexane Elimination Capacity following acclimation period with methanol across all four TBABs.

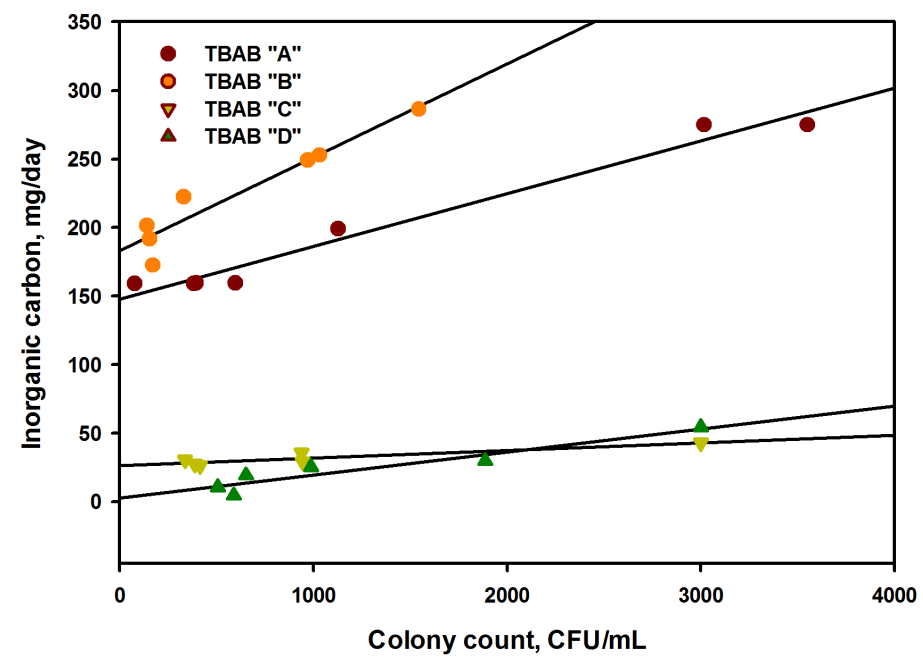

Figure 7. Inorganic carbon (mg/day) detected in each of the TBABs in comparison to viable biomass $(\mathrm{cfu} / \mathrm{ml})$.

viable biomass. It also confirms previous findings that TBABs $A$ and $B$ were functioning at higher removal efficiencies than TBABs $C$ and D.

Lastly, as with trends in species counts, overall VSS followed similar patterns in TBABs which were set to similar conditions. TBABs A and B followed very similar patterns throughout the filtration period, as seen in Figure 8. In addition, ТВАВ B maintained higher VSS counts over time, which again corresponded with overall higher viable biomass concentration and higher removal efficiency and elimination capacity as compared to TBAB A. However, it should be noted that although at times VSS and colony counts in TBAB B are much higher than that of TBAB A, elimination capacity and removal efficiency of this $\mathrm{TBAB}$ do not greatly exceed that of TBAB A; this again shows that a steady state does seem to exist in these TBABs, as with most ecological systems, in which an 


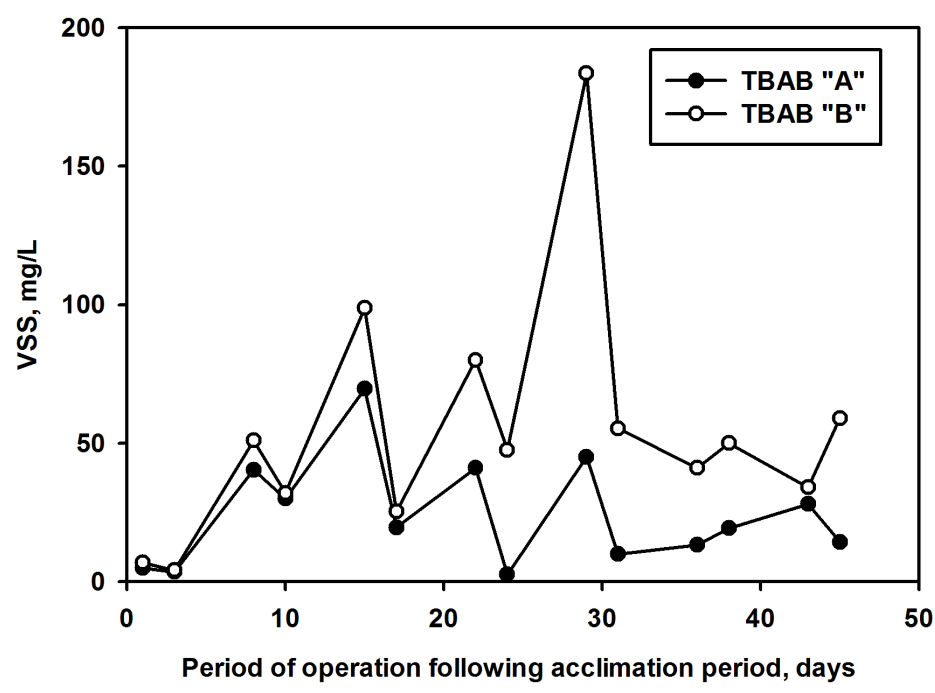

Figure 8. VSS in effluent samples from TBABs A and B against time of operation. Similar patterns are observed, all while TBAB B maintaining higher VSS.

optimum level and combination of fungal species is necessary to achieve the highest biofiltration efficiency. TBABs C and D also followed each other well, although not to the same degree of similitude of A and B, most likely due to pressure drop and biomass accumulation issues in filter $\mathrm{C}$.

\section{Conclusions}

The information attained in this study is pertinent to industries in which VOC emissions are regulated. As industries seek biofiltration as a means of efficient VOC elimination, efficiency becomes a priority in this world of profit maximization. In contrast to incineration, oxidation, and membrane filtration, biofiltration is cheaper to operate. However, issues in maintenance, reliability, and efficiency have seemed to keep biofiltration from wide-spread adoption. For this reason, this study was undertaken to gain an understanding of the ecological system within a TBAB, in hopes that this information would lead to enabling users to increase VOC removal efficiency.

Distinguishing VSS from enumeration is essential to understanding the ecology of TBABs. VSS, as it was determined here, is an inaccurate measure of viable biomass. However, as an indicator of both viable and dead biomass, VSS may indicate changes in species dominance when related inversely to plate counts. Furthermore, these changes in species dominance are essential to the functionality of the TBAB at different biomass densities. As TBABs contain their own unique ecological environments, competition and dominance will depend on the ability of the species to capture and utilize resources, namely VOCs. However, each or most of the species maintain a niche within the TBAB, and therefore, as was observed, are still present but at lower counts. Therefore, the central conclusion made through the observations is that both species type and concentration are key factors in biofiltration. As such, balancing the environment to suit the 
needs of the specific needs of the species present relies on the variety of biomass present and their subsequent population density.

Primarily, these studies along with others have sought means to decrease time for TBAB stabilization as well as VOC capture. This has been done using specific inoculums as well as the use of surfactants. However, results from this study indicate a different model. By introducing multiple species into the TBAB, flexibility is introduced into the filter. Plants which have transient VOC loading, for example, would normally have issues sustaining a higher concentration of biomass sufficient for VOC metabolism. With a TBAB as constructed in this study, one with a consortium of organisms would be able to adapt to these varying conditions, and in this specific case, fungi producing hyphae would be selected for and would continue to maximize VOC elimination capacity within the TBAB. The converse is certainly true. Therefore, if the TBAB utilizes multiple species, multiple changes in $\mathrm{TBAB}$ conditions will not limit elimination capacity, but rather provide it with natural means to accommodate these changes.

\section{References}

[1] Lanska, D.J. (1999) Limitations of Occupational Air Contaminant Standards, as Exemplified by the Neurotoxin N-hexane. Journal of Public Health Policy, 20, 441-458. https://doi.org/10.2307/3343130

[2] Costa, L.G. (1996) Biomarker Research in Neurotoxicology: The Role of Mechanistic Studies to Bridge the Gap between the Laboratory and Epidemiological Investigations. Environmental Health Perspectives, 104, 55-67. https://doi.org/10.1289/ehp.96104s155

[3] Graham, D.G., et al. (1995) Pathogenetic Studies of Hexane and Carbon Disulfide Neurotoxicity. Critical Reviews in Toxicology, 25, 91-112. https://doi.org/10.3109/10408449509021609

[4] Matsuoka, M. (2007) Neurotoxicity of Organic Solvents-Recent Findings. Brain and Nerve, 59, 591-596.

[5] Bebarta, V. and De Witt, C. (2004) Miscellaneous Hydrocarbon Solvents. Clinics in Occupational and Environmental Medicine, 4, 455-479. https://doi.org/10.1016/j.coem.2004.03.004

[6] Delhoménie, M.-C. and Heitz, M. (2005) Biofiltration of Air: A Review. Critical Reviews in Biotechnology, 25, 53-72. https://doi.org/10.1080/07388550590935814

[7] Devinny, J.S., Deshusses, M.A. and Webster, T.S. (1998) Biofiltration for Air Pollution Control. CRC Press, Boca Raton.

[8] Miller, M.J. and Allen, D.G. (2005) Biodegradation of $\alpha$-Pinene in Model Biofilms in Biofilters. Environmental Science \& Technology, 39, 5856-5863. https://doi.org/10.1021/es048254y

[9] Zhu, X., et al. (2004) Effect of Substrate Henry's Constant on Biofilter Performance. Journal of the Air \& Waste Management Association, 54, 409-418. https://doi.org/10.1080/10473289.2004.10470918

[10] Aly Hassan, A. and Sorial, G. (2009) Biological Treatment of Benzene in a Controlled Trickle Bed Air Biofilter. Chemosphere, 75, 1315-1321. https://doi.org/10.1016/j.chemosphere.2009.03.008

[11] Amouric, A., et al. (2006) Study of a Hexane-Degrading Consortium in a Biofilter 
and in Liquid Culture: Biodiversity, Kinetics and Characterization of Degrading Strains. FEMS Microbiology Ecology, 55, 239-247. https://doi.org/10.1111/j.1574-6941.2005.00017.x

[12] Arriaga, S. and Revah, S. (2005) Improving Hexane Removal by Enhancing Fungal Development in a Microbial Consortium Biofilter. Biotechnology and Bioengineering, 90, 107-115. https://doi.org/10.1002/bit.20424

[13] Cai, Z., Kim, D. and Sorial, G.A. (2007) A Comparative Study in Treating Two VOC Mixtures in Trickle Bed Air Biofilters. Chemosphere, 68, 1090-1097. https://doi.org/10.1016/j.chemosphere.2007.01.068

[14] Casset, A. and de Blay, F. (2008) Health Effects of Domestic Volatile Organic Compounds. Revue des Maladies Respiratoires, 25, 475-485.

https://doi.org/10.1016/S0761-8425(08)71587-0

[15] Cicolella, A. (2008) Volatile Organic Compounds (VOC): Definition, Classification and Properties. Revue des Maladies Respiratoires, 25, 155-163. https://doi.org/10.1016/S0761-8425(08)71513-4

[16] van Groenestijn, J., Van Heiningen, W. and Kraakman, N. (2001) Biofilters Based on the Action of Fungi. Water Science \& Technology, 44, 227-232.

[17] Cheng, Z., et al. (2016) Treatment of Gaseous Toluene in Three Biofilters Inoculated with Fungi/Bacteria: Microbial Analysis, Performance and Starvation Response. Journal of Hazardous Materials, 303, 83-93. https://doi.org/10.1016/j.jhazmat.2015.10.017

[18] Estrada, J.M., et al. (2013) A Comparative Study of Fungal and Bacterial Biofiltration Treating a VOC Mixture. Journal of Hazardous Materials, 250, 190-197. https://doi.org/10.1016/j.jhazmat.2013.01.064

[19] Lebrero, R., et al. (2016) Exploring the Potential of Fungi for Methane Abatement: Performance Evaluation of a Fungal-Bacterial Biofilter. Chemosphere, 144, 97-106. https://doi.org/10.1016/j.chemosphere.2015.08.017

[20] Jeong, E., Hirai, M. and Shoda, M. (2009) Removal of Xylene by a Mixed Culture of Pseudomonas sp. NBM21 and Rhodococcus sp. BTO62 in Biofilter. Journal of Bioscience and Bioengineering, 108, 136-141. https://doi.org/10.1016/j.jbiosc.2009.03.024

[21] Jorio, H., et al. (2009) Treatment of VOCs in Biofilters Inoculated with Fungi and Microbial Consortium. Environmental Technology, 30, 477-485. https://doi.org/10.1080/09593330902778849

[22] Prenafeta-Boldú, F.X., et al. (2008) Influence of Synthetic Packing Materials on the Gas Dispersion and Biodegradation Kinetics in Fungal Air Biofilters. Applied Microbiology and Biotechnology, 79, 319-327. https://doi.org/10.1007/s00253-008-1433-2

[23] Van Groenestijn, J., et al. (2009) Performance and Population Analysis of a Non-Sterile Trickle Bed Reactor Inoculated with Caldicellulosiruptor saccharolyticus, a Thermophilic Hydrogen Producer. Biotechnology and Bioengineering, 102, 1361-1367. https://doi.org/10.1002/bit.22185

[24] Vergara-Fernández, A., Hernández, S. and Revah, S. (2008) Phenomenological Model of Fungal Biofilters for the Abatement of Hydrophobic VOCs. Biotechnology and Bioengineering, 101, 1182-1192. https://doi.org/10.1002/bit.21989

[25] Vigueras, G., et al. (2009) Hydrophobic Response of the Fungus Rhinocladiella similis in the Biofiltration with Volatile Organic Compounds with Different Polarity. Biotechnology Letters, 31, 1203-1209. https://doi.org/10.1007/s10529-009-9987-3 
[26] Aly Hassan, A. and Sorial, G.A. (2010) Removal of Benzene under Acidic Conditions in a Controlled Trickle Bed Air Biofilter. Journal of Hazardous Materials, 184, 345-349. https://doi.org/10.1016/j.jhazmat.2010.08.042

[27] Zehraoui, A., Aly Hassan, A. and Sorial, G. (2012) Effect of Methanol on the Biofiltration of N-Hexane. Journal of Hazardous Materials, 219, 176-182. https://doi.org/10.1016/j.jhazmat.2012.03.075

[28] Montgomery, J.H. (2007) Groundwater Chemicals Desk Reference. CRC Press, Boca Raton.

[29] USEPA (2014) Superfund Chemical Data Matrix (SCDM). http://www.epa.gov/superfund/sites/npl/hrsres/tools/scdm.htm

[30] Verschueren, K. (2001) Handbook of Environmental Data on Organic Chemicals. Environmental Protection Magazine Series. John Wiley and Sons Inc., New York, 2391.

[31] APHA (2005) Standard Methods for the Examination of Water and Wastewater. American Public Health Association, Washington DC.

[32] Aly Hassan, A. and Sorial, G.A. (2010) A Comparative Study for Destruction of n-Hexane in Trickle Bed Air Biofilters. Chemical Engineering Journal, 162, 227-233. https://doi.org/10.1016/j.cej.2010.05.034

[33] St-Germain, G. and Summerbell, R. (1996) Identifying Filamentous Fungi: A Clinical Laboratory Handbook. Star Publishing Company, Singapore.

[34] Larone, D.H. (1993) Medically Important Fungi: A Guide to Identification. American Society for Microbiology. 